\title{
In situ Surface Topography of Laser Powder Bed Fusion Using Fringe Projection
}

\author{
Bin Zhang ${ }^{1}$, John Ziegert ${ }^{1}$, Faramarz Farahi ${ }^{1}$ and Angela Davies ${ }^{1}$ \\ ${ }^{1}$ The University of North Carolina at Charlotte, Charlotte, NC 28223, USA
}

\begin{abstract}
Being able to characterize the process signatures of powder bed based additive manufacturing process is key to improving the product quality. This paper demonstrates the implementation of a digital fringe projection technique to measure surface topography of the powder bed layers during the fabrication. We focus on developing the metrology tool and observing the types of information that can be extracted from such topographical data. The performance of the system is demonstrated with selected in situ measurements. Experimental results show this system is capable of measuring powder bed signatures including the powder layer flatness, surface texture, the average height drop of the fused regions, characteristic length scales on the surface, and splatter drop location and dimension.
\end{abstract}

Keywords: Additive manufacturing; fringe projection; powder bed fusion; in situ metrology; surface topography.

\section{Introduction}

Metal additive manufacturing has been developing rapidly over the past few decades. The quality of parts generated by the metal additive process has improved dramatically, but has reached a limit using the trial and error approach. The key to overcome this limit is in situ monitoring of the process signatures [1]. In situ measurements will add to our knowledge about the physics behind additive processes and expedite process development that leads to improved material and structural properties. Real-time monitoring also allows for possible feedback control to compensate or correct detected errors, or abort continued construction of defective parts.

The majority of research on in situ metrology of Laser Powder Bed Fusion (LPBF) additive processes focuses on two types of sensors - thermographic sensors and high-resolution imaging sensors. Thermographic sensors are useful for on-line monitoring of temperature related signatures, including real time melt pool dimensions, temperature profile, temperature history, as well as other factors [2-5]. High resolution imaging sensors are mostly used for evaluation of geometric features and identification of defects in each build layer [6,7]. Both of these sensors are limited to two dimensions. Investigations on in situ measurement of layer-wised surface topographies is limited, presumably because the measurement is challenging and directly-applicable sensors are not available. Although modern optical profilers, such as white light interferometers and confocal microscopes, can produce high-resolution surface measurements, their short working distance (millimeter scale) do not allow for integration into the build chamber of typical LPBF machines. More importantly, the general measurement specifications, namely a millimeter scale vertical dynamic range over a field of view on the order of one hundred millimeters, are challenging for many measurement approaches. Fringe projection is a viable solution, being well suited to measure an areal profile of a mid-sized object [8]. The flexible working distance of a fringe projection system is one of the biggest advantages, allowing it to be mounted far from the powder bed, therefore it doesn't conflict with the fabrication process. Commercial fringe projection systems are available, but none meet the height resolution requirements nor are geometrically optimized for in situ metrology.

This paper demonstrates a custom-designed fringe projection system for in situ metrology of LPBF processes. Construction of the system is described in detail in Section 2. Section 3 shows selected in situ surface topographies to demonstrate the performance of the developed system. 


\section{Fringe Projection System}

\subsection{Hardware Development}

The main components of a fringe projection system are a projector and a camera. The projector can produce structured light patterns (e.g. a sequence of sinusoidally varying intensity patterns) on the surface of the object, while the camera captures these patterns from a different viewing angle. The projected fringes appear deformed due to local height variations of the object surface. The deformed fringes captured by the camera are then reconstructed to obtain the 3D shape of the object. This study of in situ metrology is conducted on the testbed structure of the LPBF machine designed and created by the Edison Welding Institute. The setup of the fringe projection system on this machine chamber is shown in Figure 1.

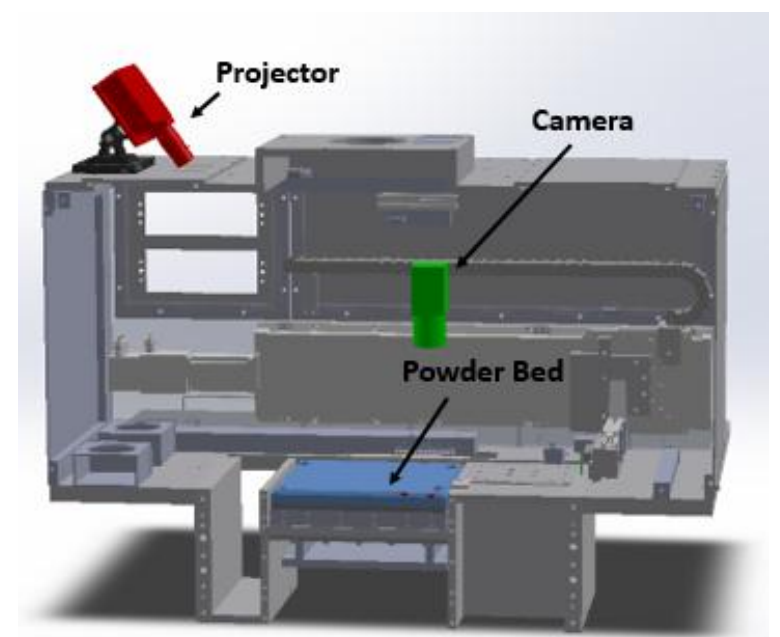

Figure 1. The setup of digital fringe projection system on the LPBF machine (some parts of the machine are hidden for clear visualization)

Inspection of the surface topography of metal LPBF samples suggests that system lateral resolution better than 10 micrometers is desirable. To achieve this, a machine vision camera (PointGray Flea3, resolution 4096×2160 pixels) with a $50 \mathrm{~mm}$ lens (Edmond Optics Part No. 86574) for high-resolution imaging of the build process was used. This camera is located about $200 \mathrm{~mm}$ above the powder bed, providing a field of view of about $28 \times 15 \mathrm{~mm}$. To achieve a micrometer-scale height measurement capability, a small effective wavelength is required. Note that effective wavelength is not the wavelength of light source, but a function of the fringe pitch and the projection angle. The phase resolution of phase measuring techniques can be as low as $0.1 \%$, meaning the height resolution of a phase-measuring fringe projection system can be as low as $0.1 \%$ of the effective wavelength. That is to say, the desired effective wavelength is below $1 \mathrm{~mm} /$ cycle for a desired height resolution. This presents a challenge when choosing the projector image size and resolution. The Nyquist sampling theorem requires the minimum sampling rate for a sine wave to be two pixels per cycle, and added noise considerations suggest the use of at least 10 pixels per cycle. Most commercial projectors have a pixel footprint of about a half millimeter, which limits the effective wavelength to above $5 \mathrm{~mm} / \mathrm{cycle}$ (if angle between projector and camera is $45^{\circ}$ ). Taking the limitation of phase deviation (1/1000 wave) into account, this effective wavelength will not meet the measurement requirements. We overcome this limit with a customized projection lens in configuration with a commercial DLP projector (Vivitek Q5, resolution $1280 \times 800$ pixels). The projector is mounted on top of the chamber, illuminating the powder bed at an angle of approximately $35^{\circ}$ through an anti-reflection coated window. The distance between projector and powder bed is about $600 \mathrm{~mm}$. The customized projection lens system creates a very small projected image (about $45 \times 28 \mathrm{~mm}$ ) that no commercial projector can produce. This small image allows us to generate dense fringes as small as $0.35 \mathrm{~mm} /$ cycle to achieve the desired height resolution. 


\subsection{Phase Shifting Algorithm}

The phase shifting algorithm is the core of the fringe projection technique. Here we use the least square phase shifting algorithm [9]. It utilizes $\mathrm{N}$ frames of sinusoidal patterns whose phases are shifted $2 \pi / \mathrm{N}$ from one frame to the next. This algorithm is optimized to minimize the phase error and its accuracy can generally be improved by increasing the number of phase steps. When operating the digital fringe projection system, a sequence of sinusoidal fringe patterns are projected on the object surface. The irradiance distribution of the patterns are expressed as,

$$
I_{i}(x, y)=I_{0}\left[1+\cos \left(\frac{2 \pi x}{p}+\delta_{i}\right)\right]
$$

where $i$ indiates the $i^{\text {th }}$ frame, $I_{0}$ is the irradiance modulation, $p$ is the period of the sine wave, $\delta$ is the step size which is given by

$$
\delta_{i}=\frac{i-1}{N} 2 \pi, \quad i=1, \ldots, N
$$

The fringe patterns are then captured by the camera, and the wrapped phase map is obtained by applying the phase shifting algorithm. The wrapped phase map is written as

$$
\phi(x, y)=\arctan \left(\frac{-\sum_{i=1}^{N} \sin \left(\delta_{i}\right) I_{i}(x, y)}{\sum_{i=1}^{N} \cos \left(\delta_{i}\right) I_{i}(x, y)}\right)
$$

In the real computation process, the four-quadrant inverse tangent function, $\operatorname{atan} 2(Y, X)$, is used to remove the sign ambiguity of the numerator and denominator.

\subsection{Phase Unwrapping}

The output of the atan 2 function is limited in the range of $[-\pi, \pi]$, but the real phase of the sine wave is continuous. Thus, this wrapped phase needs to be expanded from $[-\pi, \pi]$ to an extended range. This procedure is called phase unwrapping. The simplest phase unwrapping algorithm is to unfold all the phase jumps bigger than $\pi$ along the rows and columns respectively [10]. In this application, the unwrapping algorithm works well with the powder surface, but unwrapping errors occur for the measurements containing rough metal surfaces. Thus, a more robust algorithm is needed. Here, we introduce what we call a reference-guided unwrapping algorithm. This algorithm uses the reference phase map obtained from the first powder layer to unwrap the other wrapped phase maps. The conventional unwrapping algorithm is used for unwrapping of the first powder layer [9]. We then fit this unwrapped phase map to Legendre polynomials (up to the third order) and this polynomial fit becomes the unwrapped reference phase map used in the unwrapping algorithm in Equation 4 for all subsequent measurements. The algorithm can be mathematically expressed as

$$
\varphi_{u}(x, y)=\varphi_{r}(x, y)+2 \pi \cdot \text { Round }\left(\frac{\varphi_{r e f}(x, y)-\varphi_{r}(x, y)}{2 \pi}\right)
$$

where $\varphi_{u}(x, y)$ is the unwrapped phase, $\varphi_{r}(x, y)$ is the wrapped phase, $\varphi_{r e f}(x, y)$ is the reference map, and the operator "Round ( )" means rounding to an integer. This method is valid because all the measurements are taken at around the same height level, and the height variation of the surface is relatively small. One big advantage of this algorithm is that the phase unwrapping is achieved at each pixel such that unwrapping errors will not propagate along the pixels arrays. 
The unwrapped phase map contains both carrier phase and object phase. The carrier phase is a chirped sinusoidal wave, where the chirping is a result of the camera/projection angle. The reference phase map is also used as the carrier phase map in our algorithm. This means the reference phase maps are subtracted from the unwrapped maps, leaving only the phase information from the object that is of interest. By using the reference phase map in this way in all subsequent measurements, the height in all the topographies are relative to that of the first powder layer.

\subsection{Calibration}

The fringe projection system needs to be calibrated in order to achieve high-precision measurements. The goal of calibration is to achieve a phase-to-height conversion in the vertical direction and a pixel-to-millimeter conversion in the lateral directions. The first procedure is called, $\mathrm{z}$ calibration or effective wavelength calibration [11], and the second one is called $x-y$ calibration or lateral calibration. The effective wavelength varies over the field of view due to the change of projector magnification over the projection angle, and approximating this as a constant over the field of view can lead to a large systematic error. This problem can be solved if the effective wavelength is calculated as a function of image pixel coordinates.

\subsubsection{Effective Wavelength Calibration}

In the effective wavelength calibration, a coated piece of float glass is used as a diffuse flat surface. First, the phase shifting and phase unwrapping processes are used to obtain the unwrapped phase maps of the flat surface. Next, this step is repeated as the glass flat is moved to 10 different height positions as indicated by a separate external displacement gauge. In doing so, the unwrapped phases at each pixel can be related to the height values using a linear scaling factor, the effective wavelength, given by

$$
\lambda_{e f f}=\frac{2 \pi}{\Delta \phi / \Delta h}
$$

where $\Delta \phi$ (in radian) and $\Delta h$ (in millimeter) are the phase and height change between each height step for each pixel. In the experiment, the build platform moves from $-0.5 \mathrm{~mm}$ to $+0.5 \mathrm{~mm}$ around the nominal height of the powder layer. The resulting effective wavelength map is shown in Figure 2.

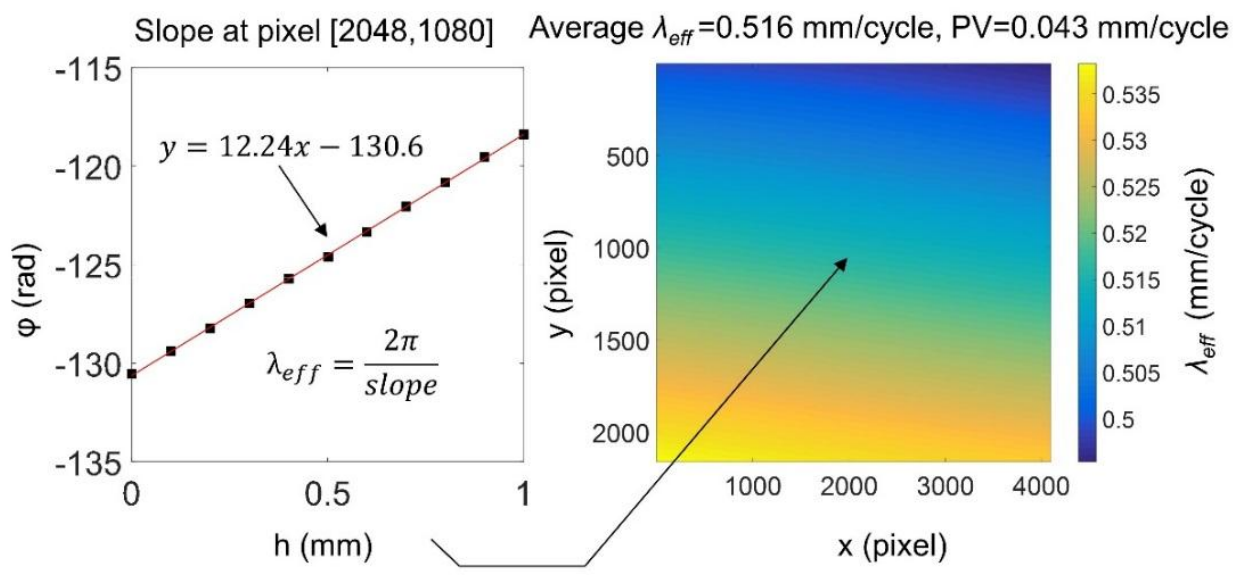

Figure 2: Effective wavelength map. Left plot shows the calibration data at the center pixel of the effective wavelength map.

Finally, the height map is obtained by multiplying the remaining phase map by the effective wavelength map.

$$
z(x, y)=\lambda_{e f f}(x, y) \cdot \phi(x, y) / 2 \pi
$$

\subsubsection{Pixel-to-millimeter Calibration}


Conventionally, lateral calibration is achieved by camera calibration [12]. Here a straight-forward pixel-tomillimeter conversion procedure is implemented using an externally-calibrated grid plate artifact $(5 \times 3$ grid with spacing $6.5 \mathrm{~mm}$ ). First, the image of the grid plate is acquired at the reference height level as shown in Figure 3 . Then the grid pitch in pixels is determined. For this setup we measured the grid pitch in both $\mathrm{x}$ and $\mathrm{y}$ directions to be around 956 pixels. In doing so, the relation between the image pixel and the real distance (in millimeters) of the grid pitch is established. Finally, the height map is converted from pixel space to millimeter space by multiplying the pixel-unit height map by the scaling factor $(6.8 \mu \mathrm{m}$ per pixel). For the fringe projection system developed here where the optical axis of the camera is perpendicular to the powder bed, this method of lateral calibration is sufficient. For more general cases where the camera is at an arbitrary angle to the measurement plane, a perspective correction procedure is needed. Details about lateral calibration with perspective correction can be found in our previous paper [13].

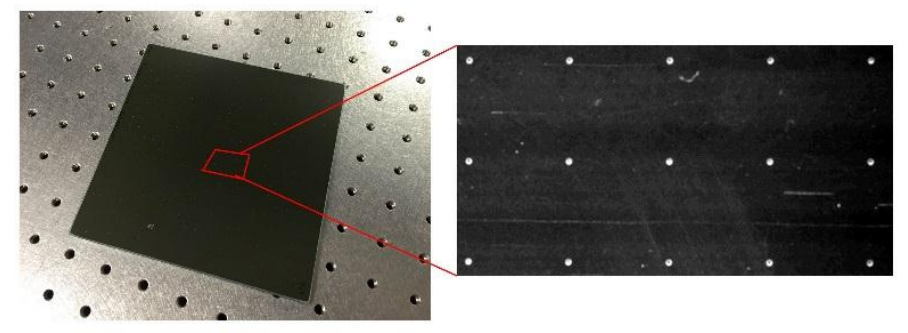

Figure 3: Lateral calibration artifact

\subsection{Practical Considerations}

One of the most common noise sources in the digital fringe projection system is the nonlinear gamma curve of the projector/camera system [6,7]. A gamma correction procedure must be applied to linearize the system input/output response curve [16] before the calibration and measurements are performed. In addition, a Fourier filtering technique is also recommended to further reduce the residual harmonic noise in the phase map.

Specular reflection with metallic surface is a problem for fringe projection systems, often causing pixel saturation, and thereby an incorrect phase determination. In our algorithm, we identify these saturated pixels, remove them, and mark them as pixels with "no data" in the final results.

For rough surfaces, "pockets" in the surface can be blocked from illumination and no light is reflected and captured by the camera, similar to the shadowing effect for non-stereo fringe projection systems. In our algorithm, the dark pixels are identified by thresholding the irradiance for all camera frames, removed and marked as black points that don't contain any height values. Although no height information is available at these locations, these markers are potentially useful for detecting defects such as pores on the fused surface.

\subsection{Measurement Capability}

The general design specifications require the metrology system to meet possess measurement capabilities which match the characteristic length scale of the process signatures, such as laser spot size $(100 \mu \mathrm{m})$, hatch distance $(90$ $\mu \mathrm{m})$ and powder layer thickness $(40 \mu \mathrm{m})$ and powder particle size. The metal powder used in this research is a Nickelbased alloy (Inconel 625) whose particle size is between $15 \mu \mathrm{m}$ and $60 \mu \mathrm{m}$. The lateral measurement capability is limited by the lateral spacing of two adjacent data points determined by the camera resolution and the field of view. For our system, the camera resolution is $4160 \times 2091$ pixels and the field of view is $28 \times 15 \mathrm{~mm}$, giving a nominal lateral spacing of $6.8 \mu \mathrm{m} / \mathrm{pixel}$.

A standard deviation of the phase in some way is usually used as a measure of the height measurement capability. In the field of digital fringe projection, it is common to evaluate the height resolution by calculating the rms phase variation over the measurement area of a flat artifact whose flatness is negligible [16-20]. This method is valid when the effective wavelength is much larger than the surface departures from flat. When the effective wavelength is very small $(<1 \mathrm{~mm} /$ cycle), the surface flatness of most measurable surfaces cannot be neglected. Alternatively one can 
evaluate the phase repeatability at each pixel, and this is the approach we take to estimate a measurement limitation. The limitation for the height measurement capability is related to the repeatability of the phase measurements and the effective wavelength by,

$$
\delta_{h}=\lambda_{e f f} \cdot \varphi_{d}
$$

where $\varphi_{d}$ is the standard uncertainty of the measured phase (in waves). The standard uncertainty is defined as the standard deviation divided by the root square of the number of measurements. The rule of thumb for a good estimation of the standard uncertainty requires a sufficiently large sample size (at least 30 measurements) according to the central limit theorem. Here we measured 30 phase maps on the flat powder layer. The standard uncertainty of the measured phase is 0.00091 waves. The single point repeatability is calculated to be $0.47 \mu \mathrm{m}$. Some of the important parameters for the measurement capability are summarized in Table 1

Table 1: Measurement Capability

\begin{tabular}{ll}
\hline Parameters & Value \\
\hline Field of view $(\mathrm{mm})$ & $28 \times 15$ \\
Lateral point spacing $(\mu \mathrm{m})$ & 6.8 \\
Average $\lambda_{\text {eff }}(\mathrm{mm} / \mathrm{cycle})$ & 0.516 \\
Standard uncertainty in phase (waves) & 0.00091 \\
Single point repeatability $(\mu \mathrm{m})$ & 0.47
\end{tabular}

\section{Results and Discussion}

This section demonstrates aspects of the LPBF process that can be suited with our fringe projection system by presenting selected in situ measurements. Because our system is in development we have not yet attempted to analyze the LPBF process characteristics based on the measurements but we believe that the measurement results show promise for such analysis. To demonstrate a typical measurement, a $6 \times 6 \mathrm{~mm}$ square pillar is built with the nominal process parameters, i.e. laser power $290 \mathrm{~W}$, scan speed $960 \mathrm{~mm} / \mathrm{s}$, hatch distance $0.09 \mathrm{~mm}$ and powder layer thickness $40 \mu \mathrm{m}$. The laser scans back and forth along the recoating direction without rescanning the contour of the square as shown in Figure 4 (a). An alternating scan strategy is used, meaning the scan direction is perpendicular between the two adjacent layers. The powder surface is measured with an average $0.52 \mathrm{~mm} /$ cycle effective wavelength, and an example of the fringe patterns is shown in Figure 4 (b). The height map of this surface is shown in Figure 4 (c). Although a small percentage of data drop-outs (black and white points which represent low intensity and saturated pixels respectively) exist, the height map provides rich information about the fusion process. Firstly, the boundary of the fused square can be very well defined by a narrow groove. This distinct structure is not easily extracted from digital images (photographs) of the surface and is potentially useful for applications such as edge detection and geometric accuracy evaluation. Secondly, the average height of the fused surface is lower than that of the unfused surface as a result of powder solidification. This can be observed as an average color change between the fused and the unfused regions. The average height difference between the fused and unfused surface can also be calculated and tracked by layers. This will be discussed later in this section. Thirdly, many detailed features are observed in the height map of the fused region, such as the abrupt changes in the elevation at the edges, and the height variation within the fused region. Small higher spots are apparent in the height map, particularly noticeable in the unfused regions, and presumably are the splatter drops formed during the laser fusion process. Splatter drops are expected on both fused and unfused regions. Although impact of spatter drops on the final product quality is unclear, the ability to detect them may provide useful information for process control and yield assessment. 

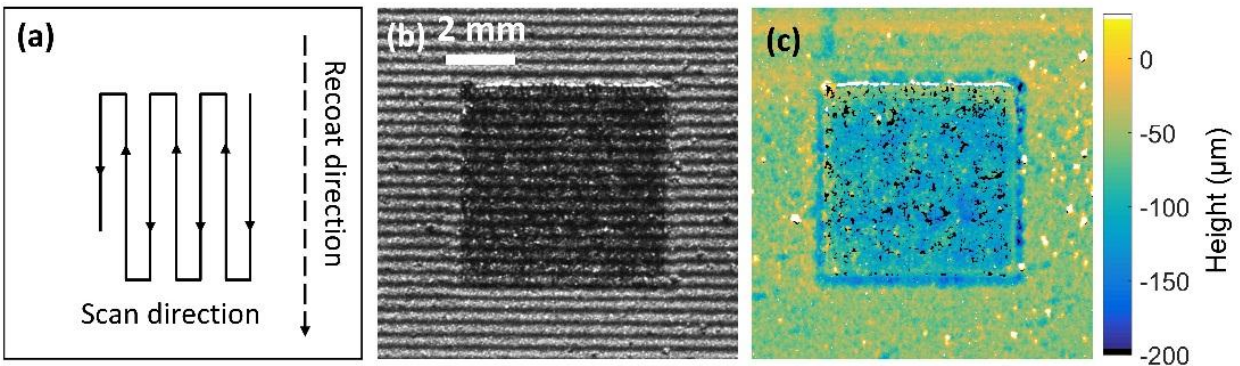

Figure 4: (Color online) (a) Schematics of laser scan path and recoating direction (b) Photograph of deformed fringe pattern on the fused powder surface on the $18^{\text {th }}$ layer and (c) the corresponding fringe projection measurement of the surface topography. Black and white points in (c) are data drop-outs caused by shadowing and camera saturation respectively.

The build process is monitored by taking two measurements for each layer. The first one is after the new layer of powder is spread on the powder bed, and this measurement monitors the flatness and height consistency of the powder layer. The second measurement is taken after laser fusion. These measurements provide rich information about the effects of the process. Figure 5 shows the in situ height maps of the powder bed at Layers 2, 4, and 6. The color bar is fixed to range from -0.2 to $0.1 \mathrm{~mm}$ for easy comparison between the height maps. Several interesting aspects of the height maps before fusion (left column) are evident. "Waviness" can be observed on the powder layers. For example, the two small arrows next to Layer 2 in Figure 5 show powder surface waves with a peak-to-valley height of about 28 $\mu \mathrm{m}$. This is assumed due to vertical motions of recoating blade as it goes across the build area. Fused metal can also be exposed above the powder surface if the local height of the build is over the powder level. This can be observed as the saturated points (white points) along the edge of the fused region. The average height of the powder is a useful value, representing the average distance from the measurement plane to the projector/camera fringe projection system. Thermal growth of the chamber or drift in the build platform position can cause this distance to change.

For the height maps after fusion (right column in Figure 5), the general features discussed above continue - a narrow groove is around the boundary of the fused area, the edges of the fused surface are slightly above the average powder height level, and the average height of the fused region is significantly below the powder level. The height maps also show that the fused surface changes with layer number. For example, the edge roughness reduces as the layer number increases. The height variation of the fused region also appears to decrease as the build layer accumulates, while the average depth (indicated by color) of the fused area increases with layer number. 

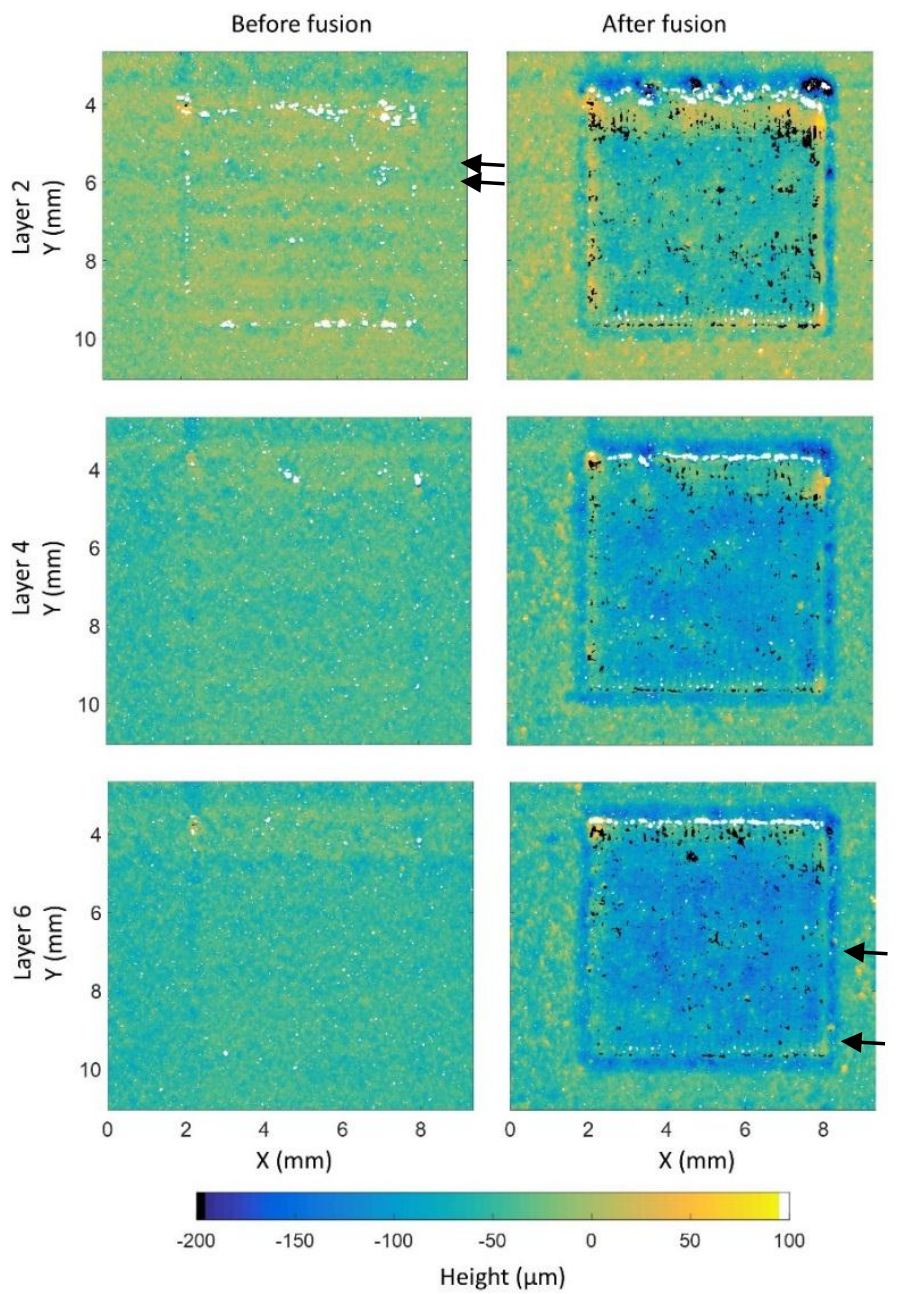

Figure 5: Height maps of powder bed before and after laser fusion measured on every other layers

The surface produced by LPBF process is expected to have an oriented linear structure along the laser scanning direction [6], and Fourier analysis of the height maps can be used to monitor such structures. As an example, the onedimensional Fourier transform of each of ten lines around the center and edge of the fused region, indicated by the tick marks at the $6^{\text {th }}$ fused layer shown in Figure 5, are averaged together. The results are shown in Figure 6. The average Fourier transform at the bottom region shows a signature peak at the spatial frequency of $5.6 \mathrm{~mm}^{-1}$, which is the frequency corresponding to twice the hatch distance. There is no observable peak at the laser raster frequency, i.e. $11.5 \mathrm{~mm}^{-1}$, but this could be because this high spatial frequency is not captured well by the measurement. Although the instrument transfer function has yet to be characterized, it is reasonable to expect that measurements of spatial frequencies approaching 0.1 Nyquist are significantly attenuated [21]. Tenth Nyquist is indicated in the figure, and we see that the $11.5 \mathrm{~mm}^{-1}$ laser scan spatial frequency (1/hatch distance) is to the right. Interestingly, the Fourier spectrum in the middle of the fused region does not show any strong peaks. Ex-situ measurements of the fused regions with a confocal microscope (with much higher spatial resolution and a 0.1 Nyquist frequency of $77 \mathrm{~mm}^{-1}$ ) confirms this claim. The high resolution confocal measurement shows that the Fourier component corresponding to twice the hatch distance is stronger than the component corresponding to the hatch distance, suggesting an asymmetry in the fusion process when scanning toward the edge versus away from the edge. 

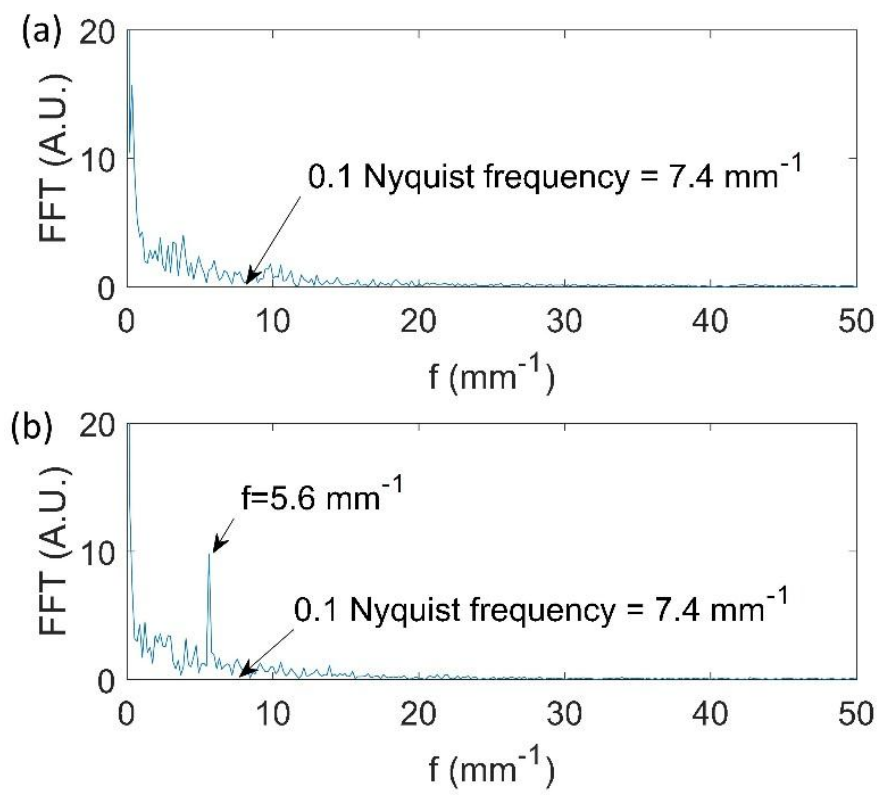

Figure 6: An average of 10 FFTs of line traces at center (a) and bottom (b) at Layer 6 as indicated by tick marks in Figure 5.

The fusion process is expected to reach a stable state after a few layers of fusion. This can be monitored by tracking the average height drop between the fused and unfused regions. These two regions are defined well inside and outside of the fused boundary, as indicated in Figure 7. The average height drop is taken to be the average height of the unfused region minus that of the fused region.

Figure 8 shows the average height drop as a function of layer number. A rapid decrease of the average height is seen for the first few layers, and then it converges to a constant value. The error bars in the graph are the standard deviation of the average height and are very small. A simple model is constructed to predict the trend of the average height drop. This model considers the powder shrinkage to be a constant factor during the process. The amount of height drop depends on the powder layer thickness and the shrinkage ratio. The shrinkage ratio, $\beta$, is defined as the ratio of powder density to the metal density. The average height drop for each layer, $h_{n}$, can be expressed as

$$
\begin{gathered}
h_{1}=-t \cdot \alpha \\
h_{2}=-\left(t+h_{1}\right) \cdot \alpha \\
\vdots \\
h_{n}=-\left(t+h_{n-1}\right) \cdot \alpha(8)
\end{gathered}
$$

where $n$ is the layer number, $t$ is the powder layer thickness and $\alpha=1-\beta$. The negative sign means the average height of the fused surface is below the powder level. This series represents a geometric progression, so the average height drop can written as

$$
h_{n}=-t \alpha \cdot\left(\frac{1-\alpha^{n}}{1-\alpha}\right)
$$

Notice this equation converges to a constant value. Interestingly, the average height drop at the stable state can be bigger than the powder layer thickness, if the shrinkage ratio $\beta$ is smaller than 0.5 . 


$$
\lim _{n \rightarrow \infty} h_{n}=-t \cdot \frac{\alpha}{\beta}
$$

A least square approach is used to determine the best-fit value for $\alpha$ using the $40 \mu \mathrm{m}$ powder thickness value as a constant [22]. The best fit indicates a value for $\alpha$ of 0.59 , representing a powder-to-metal density ratio of $\beta=0.41$. Inserting this value of $\alpha$ into Equation 10, the steady-state height drop is estimated to be $58 \mu$ m. The model with these parameters, Equation 9, is also plotted in Figure 8.

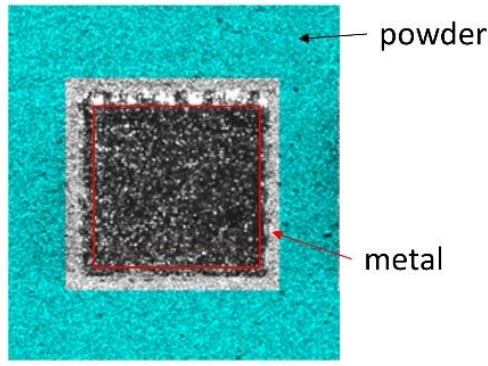

Figure 7: Photograph of a fused layer. The powder region, indicated by the light green area, is used to calculate the average height of the powder region, and the region inside the red box is used for the average height of the fused region.

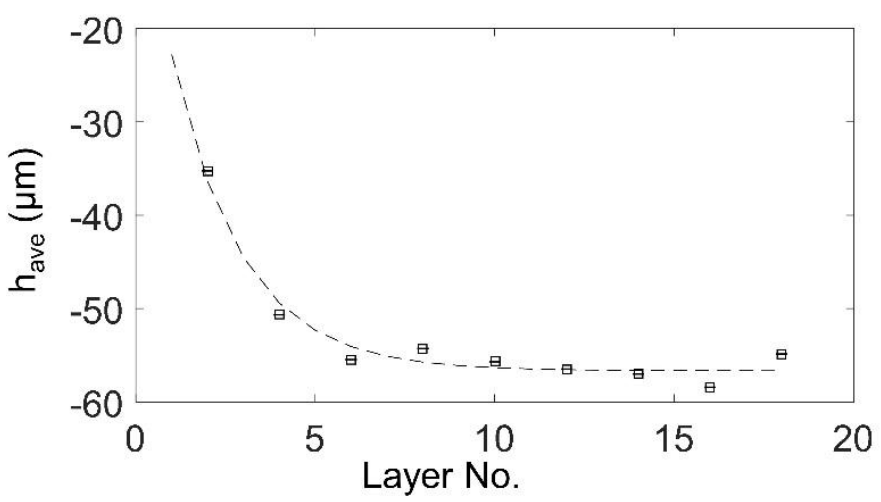

Figure 8: Average height difference between metal and powder as a function of layer number. The dashed line is the best fit of Equation 9 to the measurement data.

As mentioned in Section 2.5, the black points on the height map are data drop-out due to low signal, presumably from porous structures where there is little reflected light received by the camera due to shadowing or trapping. There is a different percentage of black points when various laser powers are used to fuse the powder. Figure 9 shows the height map of the three square pillars fused with laser powers of $350 \mathrm{~W}, 290 \mathrm{~W}$ and $230 \mathrm{~W}$ from left to right, while other process parameters (scan speed $960 \mathrm{~mm} / \mathrm{s}$, hatch distance $0.09 \mathrm{~mm}$ and powder layer thickness $40 \mu \mathrm{m}$ ) are the same. It can be seen from Figure 9 the amount of data drop-out increases as the laser power decreases. It is known that higher laser power usually creates smoother surfaces, while low laser power produces rough and porous surfaces [23]. This agrees with our observation. The percentage of data drop-out for the three squares are $1.85 \%, 9.09 \%$ and $25.39 \%$ left to right. 


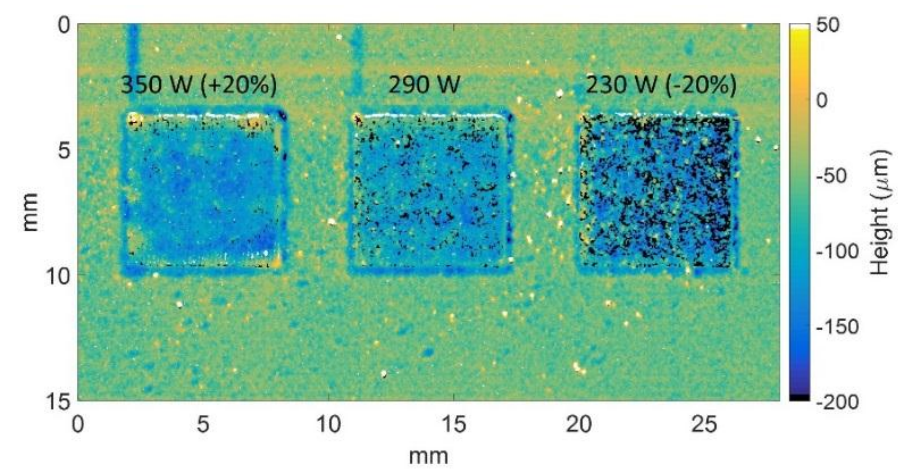

Figure 9: Image of powder bed surface (top) and height map taken after laser fusion (bottom) at the $18^{\text {th }}$ layer

To demonstrate the ability to measure small features, a $1 \times 1 \mathrm{~mm}$ patch is cropped from the center of the left fused region in Figure 9. This small height map is shown in Figure 10. The measurement shows the fused region has over $120 \mu \mathrm{m}$ in height variation over this area. Figure 10 (b) shows a line profile of the surface along the red line indicated in Figure 10 (a). The peak-to-valley height variation along this line is about $75 \mu \mathrm{m}$.
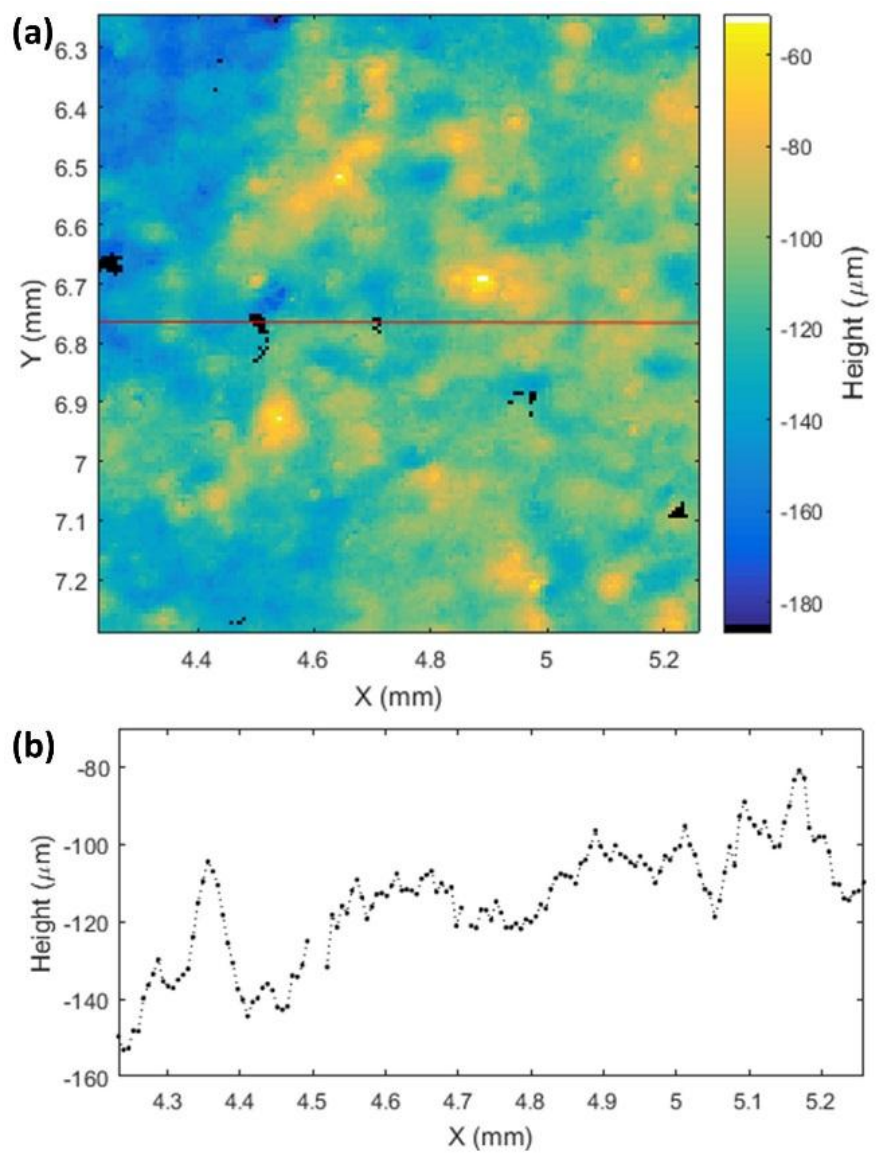

Figure 10: The height map of the central patch of the fused surface created with $350 \mathrm{~W}$ laser power at the $18^{\text {th }}$ layer (top), and the cross section profile (bottom) along the red line at $\mathrm{y}=6.77 \mathrm{~mm}$.

\section{Conclusion}

Among all in situ measurement techniques reported to date, the surface topography of the powder bed and fused metal surface has not been widely investigated. An in situ fringe projection technique for LPBF has been developed 
to enable detailed layer-by-layer surface topography measurements with the goal of increasing our understanding of the process. The development of the system has been discussed in detail. The lateral and vertical measurement capability have been characterized using metrics called lateral point spacing and single point repeatability. In situ height maps have shown useful information about the fusion process, including the powder texture, fused region height variation, characteristic length scales on the surface, and average height drop of the fused regions. The results of this study indicate that fringe projection is a viable solution for in situ surface topography metrology. Implementation of this technique will help to further understanding of the LPBF process, and provide useful feedback for future closeloop process control.

A limitation of this study is that the optimization of the projector brightness and camera exposure is still achieved by a trial and error approach. This is a nontrivial research, because the optical scattering behaves differently on the metal and powder surfaces, and the theory of fringe projection on the hybrid-material surface is not yet established. Further study regarding to this would be worthwhile.

\section{Acknowledgement}

This project is funded by Edison Welding Institute, American Makes, and National Institute of Standards and Technology.

\section{References}

[1] M. Mahesh, B. Lane, A. Donmez, S. Feng, S. Moylan, R. Fesperman, Measurement Science Needs for Realtime Control of Additive Manufacturing Powder Bed Fusion Processes, 2015. http://dx.doi.org/10.6028/NIST.IR.8036.

[2] T. Craeghs, S. Clijsters, J.-P. Kruth, F. Bechmann, M.-C. Ebert, Detection of Process Failures in Layerwise Laser Melting with Optical Process Monitoring, Phys. Procedia. 39 (2012) 753-759. doi:10.1016/j.phpro.2012.10.097.

[3] P. Lott, H. Schleifenbaum, W. Meiners, K. Wissenbach, C. Hinke, J. Bültmann, Design of an Optical system for the In Situ Process Monitoring of Selective Laser Melting (SLM), Phys. Procedia. 12 (2011) 683-690. doi:10.1016/j.phpro.2011.03.085.

[4] H. Krauss, T. Zeugner, M.F. Zaeh, Layerwise Monitoring of the Selective Laser Melting Process by Thermography, Phys. Procedia. 56 (2014) 64-71. doi:10.1016/j.phpro.2014.08.097.

[5] K. a. Mumtaz, N. Hopkinson, Selective Laser Melting of thin wall parts using pulse shaping, J. Mater. Process. Technol. 210 (2010) 279-287. doi:10.1016/j.jmatprotec.2009.09.011.

[6] J. Zur Jacobsmuhlen, S. Kleszczynski, D. Schneider, G. Witt, High resolution imaging for inspection of Laser Beam Melting systems, 2013 IEEE Int. Instrum. Meas. Technol. Conf. (2013) 707-712. doi:10.1109/I2MTC.2013.6555507.

[7] S. Kleszczynski, J. zur Jocobsmuhlen, J.T. Sehrt, Error Detection in Laser Beam Melting Systems by High Resolution Imaging, in: Twenty Third Annu. Int. Solid Free. Fabr. Symp., 2012.

[8] S.S. Gorthi, P. Rastogi, Fringe projection techniques: Whither we are?, Opt. Lasers Eng. 48 (2010) 133-140. doi:10.1016/j.optlaseng.2009.09.001.

[9] K. Creath, J. Wyant, Phase Shifting Interferometry, in: Opt. Shop Test., 1992: p. 602.

[10] K. Creath, J.C. Wyant, Moiré and Fringe Projection Techniques, in: Opt. Shop Test., 1992: p. 653.

[11] D. Purcell, A. Davies, F. Farahi, Effective wavelength calibration for moiré fringe projection, Appl. Opt. 45 (2006) 8629. doi:10.1364/AO.45.008629. 
[12] S. Zhang, Recent progresses on real-time 3D shape measurement using digital fringe projection techniques, Opt. Lasers Eng. 48 (2010) 149-158. doi:10.1016/j.optlaseng.2009.03.008.

[13] W.S. Land, B. Zhang, J. Ziegert, A. Davies, In-Situ Metrology System for Laser Powder Bed Fusion Additive Process, Procedia Manuf. 1 (2015) 393-403. doi:10.1016/j.promfg.2015.09.047.

[14] T. Hoang, B. Pan, D. Nguyen, Z. Wang, Generic gamma correction for accuracy enhancement in fringeprojection profilometry, 35 (2010) 1992-1994.

[15] T.M. Hoang, Simple Gamma Correction for Fringe Projection Profilometry System, 1428 (2010) 4503.

[16] H. Guo, H. He, M. Chen, Gamma correction for digital fringe projection profilometry., Appl. Opt. 43 (2004) 2906-14. http://www.ncbi.nlm.nih.gov/pubmed/15143816.

[17] K. Leonhardt, U. Droste, H.J. Tiziani, Microshape and rough-surface analysis by fringe projection., Appl. Opt. 33 (1994) 7477-7488. doi:10.1364/AO.33.007477.

[18] Y. Fu, Y. Wang, W. Wang, J. Wu, Least-squares calibration method for fringe projection profilometry with some practical considerations, Optik (Stuttg). 124 (2013) 4041-4045. doi:10.1016/j.ijleo.2012.12.041.

[19] S. Wang, C.J. Tay, C. Quan, H.M. Shang, Investigation of membrane deformation by a fringe projection method., Appl. Opt. 41 (2002) 101-7. doi:10.1364/AO.41.000101.

[20] X. Su, Q. Zhang, Dynamic 3-D shape measurement method: A review, Opt. Lasers Eng. 48 (2010) 191-204. doi:10.1016/j.optlaseng.2009.03.012.

[21] X. Colonna De Lega, P. de Groot, Lateral resolution and instrument transfer function as criteria for selecting surface metrology instruments, Imaging Appl. Opt. Tech. Dig. (2012) 1-3. doi:10.1364/OFT.2012.OTu1D.4.

[22] D.K.R. Philip R. Bevington, Data Reduction and Error Analysis for Physical Sciences, 3rd ed., McGrw-Hill, 2002.

[23] S. Kleszczynski, J. Jacobsmühlen, J.T. Sehrt, G. Witt, Mechanical Properties of Laser Beam Melting, (2013) $153-166$. 